\title{
Universiteit
}

Leiden

The Netherlands

\section{Maximally entangled mixed-state generation via local operations}

Aiello, A.; Puentes, G.; Voigt, D.; Woerdman, J.P.

\section{Citation}

Aiello, A., Puentes, G., Voigt, D., \& Woerdman, J. P. (2007). Maximally entangled mixed-state generation via local operations. Physical Review A, 75, 062118.

doi:10.1103/PhysRevA.75.062118

Version: $\quad$ Not Applicable (or Unknown)

License: $\quad$ Leiden University Non-exclusive license

Downloaded from: https://hdl.handle.net/1887/61252

Note: To cite this publication please use the final published version (if applicable). 


\title{
Maximally entangled mixed-state generation via local operations
}

\author{
A. Aiello, G. Puentes, D. Voigt, and J. P. Woerdman \\ Huygens Laboratory, Leiden University, P.O. Box 9504, 2300 RA Leiden, The Netherlands
}

(Received 18 July 2006; published 28 June 2007)

\begin{abstract}
We present a general theoretical method to generate maximally entangled mixed states of a pair of photons initially prepared in the singlet polarization state. This method requires only local operations upon a single photon of the pair and exploits spatial degrees of freedom to induce decoherence. We report also experimental confirmation of these theoretical results.
\end{abstract}

DOI: 10.1103/PhysRevA.75.062118

PACS number(s): 03.65.Ud, 03.67.Mn, 42.25.Ja

\section{INTRODUCTION}

Entanglement is perhaps the most puzzling feature of quantum mechanics and in the last two decades it became the key resource in quantum-information processing [1]. Entangled qubits prepared in pure, maximally entangled states are required by many quantum-information processes. However, in the mundane world, a pure maximally entangled state is an idealization such as, e.g., a plane wave in classical optics. In fact, the interaction of qubits with the environment leads to decoherence that may cause a pure entangled state to become less pure (mixed) and less entangled. Thus, any realistic quantum-communication and computation protocol must cope with entangled mixed states and it is desirable to attain the maximum amount of entanglement for a given degree of mixedness. States that fulfill this condition are called maximally entangled mixed states (MEMSs) and recently they have been the subject of several papers (see, e.g., [2,3] and references therein). In this article we propose a method to create MEMSs from a pair of photons initially prepared in the singlet polarization state.

Peters et al. [2] were the first to achieve MEMSs using photon pairs from spontaneous parametric down-conversion (SPDC). They induced decoherence in SPDC pairs initially prepared in a pure entangled state by coupling polarization and time degrees of freedom of the photons. At the same time, a somewhat different scheme was used by De Barbieri et al. [3] who instead used the spatial degrees of freedom of SPDC photons to induce decoherence. However, both methods require operations on both photons of the SPDC pair. On the contrary, our technique has the advantage to require only local operations upon one of the two photons.

This article is structured as follows: In the first part of Sec. II we show the relation existing between a one-qubit quantum map and a classical-optics setup on the laboratory bench. In the second part of Sec. II, we exploit this knowledge to design a simple linear-optical setup to generate MEMSs from a pair of photons via local operations and postselection. Then, in Sec. III we provide an experimental demonstration of our method, using entangled photons from parametric down-conversion. Finally, we draw our conclusions in Sec. IV.

\section{THEORY}

We begin by giving a brief description of the connection between classical polarization optics and quantum mechanics of qubits, as recently put forward by several authors $[4,5]$. Most textbooks on classical optics introduce the concept of polarized and unpolarized light with the help of the Jones and Stokes-Mueller calculi, respectively [6]. In these calculi, the description of classical polarization of light is formally identical to the quantum description of pure and mixed states of two-level systems, respectively [7]. Mathematically speaking, there is an isomorphism between the quantum density matrix $\rho$ describing a qubit and the classical coherency matrix $J$ [8] describing polarization of a beam of light: $\rho$ $\sim J / \operatorname{Tr} J$, where $J$ is a Hermitean, positive-semidefinite 2 $\times 2$ matrix, as is $\rho$. A classical linear optical process (such as, e.g., the passage of a beam of light through an optical device) can be described by a $4 \times 4$ complex-valued matrix $\mathcal{M}$ such that $\left(J_{\text {out }}\right)_{i j}=\mathcal{M}_{i j, k l}\left(J_{\text {in }}\right)_{k l}$, where, from now on, we adopt the convention that summation over repeated Latin indices is understood. Moreover, we assume that all Latin indices $i, j, k, l, m, n, \ldots$ take the values 0 and 1 , while Greek indices $\alpha, \beta, \ldots$ take the values $0,1,2,3$. In polarization optics one usually deals with the real-valued Mueller matrix $M$ which is connected to $\mathcal{M}$ via a unitary transformation $\Lambda: M$ $=\Lambda^{\dagger} \mathcal{M} \Lambda$ [9]. The matrix $M$ is often written as [10]

$$
M=\left(\begin{array}{cc}
m_{00} & \mathbf{d}^{T} \\
\mathbf{p} & W
\end{array}\right),
$$

where $(\mathbf{p}, \mathbf{d}) \in \mathbb{R}^{3}$ are known as the polarizance vector and the diattenuation vector (superscript $T$ indicates transposition), respectively. Note that $\mathbf{d}$ is nonzero only for dichroic media: namely, media that induce polarization-dependent losses (PDLs) [6]. $W$ is a $3 \times 3$ real-valued matrix. It should be noticed that if we choose $m_{00}=1$ (this can be always done since it amounts to a trivial polarization-independent renormalization), the Mueller matrix of a nondichroic optical element $(\mathbf{d}=\mathbf{0})$ is formally identical to a nonunital, tracepreserving, one-qubit quantum map (also called a channel) [11]. If also $\mathbf{p}=\mathbf{0}$ (pure depolarizers and pure retarders [6]), then $M$ becomes identical to a unital, one-qubit channel [1]. It is not difficult to show that any linear-optical device that can be represented by $\mathcal{M}$ (or $M$ ) can also be described by a set of at most four distinct optical elements in parallel as $\mathcal{M}=\Sigma_{\alpha} \lambda_{\alpha} T_{\alpha} \otimes T_{\alpha}^{*}$, where the four $2 \times 2$ Jones matrices $T_{\alpha}$ represent four different nondepolarizing optical elements and $\lambda_{\alpha} \geqslant 0[9,12]$. From the results above it readily follows that the most general operation that a linear optical element can perform upon a beam of light can be written as $J_{\text {in }} \rightarrow J_{\text {out }}$ 
$=\Sigma_{\alpha} \lambda_{\alpha} T_{\alpha} J_{\text {in }} T_{\alpha}^{\dagger}$. Since $\lambda_{\alpha} \geqslant 0$, the previous equation is formally identical to the Kraus form [1] of a completely positive one-qubit quantum map $\mathcal{E}$. Therefore, if a single photon encoding a polarization qubit passes through an optical device classically described by the Mueller matrix $\mathcal{M}$ $=\Sigma_{\alpha} \lambda_{\alpha} T_{\alpha} \otimes T_{\alpha}^{*}$, its initial state $\rho_{\text {in }}$ will be transformed according to $\rho_{\text {in }} \rightarrow \rho_{\text {out }} \propto \Sigma_{\alpha} \lambda_{\alpha} T_{\alpha} \rho_{\text {in }} T_{\alpha}^{\dagger}$.

Now that we have learned how to associate a quantum map to a set of at most four optical elements, we can apply this knowledge to design a simple optical scheme suitable for MEMS production. Suppose to have two qubits (encoded in the polarization degrees of freedom of two SPDC photonssay, $A$ and $B$ ), initially prepared in the state $\rho: \rho$ $=\rho_{i j, k l}|i j\rangle\left\langle k l\left|\doteq \rho_{i k, j l}^{R}\right| i\right\rangle\langle k|\otimes| j\rangle\langle l|$. The superscript $R$ indicates reshuffling [13] of the indices: $\rho_{i k, j l}^{R} \equiv \rho_{i j, k l}$. Following Ziman and Bužek [14] we assume that $\rho$ is transformed under the action of the most general local (that is, acting upon a single qubit) linear map $\mathcal{E} \otimes \mathcal{I}$ into the state

$$
\rho_{\mathcal{E}}=\mathcal{E} \otimes \mathcal{I}[\rho] \propto \sum_{\alpha=0}^{3} \lambda_{\alpha} T_{\alpha} \otimes I \rho T_{\alpha}^{\dagger} \otimes I .
$$

By writing explicitly Eq. (2) in the two-qubit basis $\{|i j\rangle$ $\equiv|i\rangle \otimes|j\rangle\}, \quad$ it is straightforward to obtain $\left(\rho_{\mathcal{E}}\right)_{i j, k l}$ $\propto \Sigma_{\alpha} \lambda_{\alpha} \rho_{m n, j l}^{R}\left(T_{\alpha}\right)_{i m}\left(T_{\alpha}^{*}\right)_{k n}$. Then, from the definition of $\mathcal{M}$ it easily follows that $\left(\rho_{\mathcal{E}}\right)_{i j, k l} \propto\left(\mathcal{M} \rho^{R}\right)_{i k, j l}$. By reshuffling $\rho_{\mathcal{E}}$, this last result can be written in matrix form as $\rho_{\mathcal{E}}^{R} \propto \mathcal{M} \rho^{R}$ which displays the very simple relation existing between the classical Mueller matrix $\mathcal{M}$ and the quantum state $\rho_{\mathcal{E}}$. Via a direct calculation, it is possible to show that if $\rho$ represents two qubits in the singlet state $\rho_{s}=\frac{1}{4}\left(I \otimes I-\sigma_{x} \otimes \sigma_{x}-\sigma_{y} \otimes \sigma_{y}\right.$ $-\sigma_{z} \otimes \sigma_{z}$ ) [15], then the proportionality symbol in the last equation above can be substituted with the equality symbol: $\rho_{\mathcal{E}}^{R}=\mathcal{M} \rho_{s}^{R}$. Note that this pleasant property is true only for the singlet state. However, if the initial state $\rho$ is different from the singlet one, then $\mathcal{M}$ must be simply renormalized by imposing $\operatorname{Tr}\left(\mathcal{M} \rho^{R}\right)=1$.

Now, suppose that we have an experimental setup producing pairs of SPDC photons in the singlet state $\rho_{s}$ and we want to transform $\rho_{s}$ into the target state $\rho_{\mathcal{T}}$ via a local map $\mathcal{T}$ $\otimes \mathcal{I}: \rho_{s} \rightarrow \rho_{\mathcal{T}}=\left(\mathcal{M}_{\mathcal{T}} \rho_{s}^{R}\right)^{R}$. All we have to do is first to invert the latter equation to obtain

$$
\mathcal{M}_{\mathcal{T}}=\rho_{\mathcal{T}}^{R}\left(\rho_{s}^{R}\right)^{-1}
$$

and then to decompose $\mathcal{M}_{\mathcal{T}}$ as $\mathcal{M}_{\mathcal{T}}=\Sigma_{\alpha} \lambda_{\alpha} T_{\alpha} \otimes T_{\alpha}^{*}$. Thus, we get the (at most four) Jones matrices $T_{\alpha}$ representing the optical elements necessary to implement the desired transformation. This is the main theoretical result of this article. Our technique is very straightforward, and we shall demonstrate its feasibility later by applying it to design an optical setup devoted to MEMS generation. However, at this moment, some caveats are in order. To make $\mathcal{M}_{\mathcal{T}}$ a physically realizable Mueller matrix, its associated matrix $H_{\mathcal{T}}$ should be positive semidefinite [16]. If this is not the case, then the transformation $\rho \rightarrow \rho_{\mathcal{T}}$ cannot be implemented via local operations. For example, it is easy to see that if the initial state is a Werner state $\rho_{W}=p \rho_{s}+\frac{1-p}{4} I(0 \leqslant p \leqslant 1)$ and the target state is the singlet $\rho_{\mathcal{T}}=\rho_{s}$, then such an operation (known as concentration [17]) cannot be physically implemented by a local setup since $H_{\mathcal{T}}$ has three degenerate negative eigenvalues. Another caveat comes from the no-signaling constraint. Since $\mathcal{M}_{\mathcal{T}}$ describes a local device operating only upon photon $A$, a second observer watching at photon $B$ cannot distinguish the initial state $\rho_{s}$ from the transformed state $\rho_{\mathcal{T}}$ : that is, $\rho^{B}=\operatorname{Tr}_{A}\left(\rho_{s}\right)=\operatorname{Tr}_{A}\left(\rho_{\mathcal{T}}\right)$. This condition requires the one-qubit map $\mathcal{T}$ to be trace preserving, $\Sigma_{\alpha} \lambda_{\alpha} T_{\alpha}^{\dagger} T_{\alpha}=I$. From Eq. (1), a straightforward calculation shows that such a condition cannot be fulfilled if $\mathbf{d} \neq \mathbf{0}$-that is, if the device implementing $\mathcal{T}$ contains dichroic (or PDL) elements.

PDL is important in many commonly used optical devices as polarizers, circulators, isolators, etc. [6]. Within the framework of quantum information theory, all these physical devices may be represented by " unphysical" one-qubit maps $\mathcal{T}$ that violate the no-signaling condition. This apparent paradox disappears if one allows causal classical communications between observers who actually measure and reconstruct the target state $\rho_{\mathcal{T}}$ generated by the "unphysical" local map $\mathcal{T} \otimes \mathcal{I}$ [18]. In fact, in coincidence measurements (required to reconstruct $\rho_{\mathcal{T}}$ ), classical (as opposed to quantum) signaling between the two observers is necessary to allow them to compare their own experimental results and select from the raw data the coincidence counts. In other words, a coincidence measurement post-selects only those photons that have not been absorbed by the PDL element [4].

With these caveats in mind, we come to the experimental validation of our method. We choose to generate MEMS I states [19], represented by the density matrix $\rho_{\mathrm{I}}=p\left|\phi_{+}\right\rangle\left\langle\phi_{+}\right|$ $+(1-p)|01\rangle\langle 01|$, where $\left|\phi_{+}\right\rangle=(|00\rangle+|11\rangle) / \sqrt{2}$ and $(2 / 3 \leqslant p$ $\leqslant 1)$. By varying the parameter $p$, the entanglement and mixedness of the state $\rho_{\mathrm{I}}$ change. Here, we use the linear entropy $S_{L}[20]$ and the tangle $T$-namely, the concurrence squared [21] - to quantify the degree of mixedness and of entanglement, respectively. They are defined as $S_{L}(\rho)=\frac{4}{3}\left[1-\operatorname{Tr}\left(\rho^{2}\right)\right]$ and $T(\rho)=\left[\max \left\{0, \sqrt{\lambda_{0}}-\sqrt{\lambda_{1}}-\sqrt{\lambda_{2}}-\sqrt{\lambda_{3}}\right\}\right]^{2}$, where $\lambda_{0} \geqslant \lambda_{1}$ $\geqslant \lambda_{2} \geqslant \lambda_{3} \geqslant 0$ are the eigenvalues of $\rho\left(\sigma_{y} \otimes \sigma_{y}\right) \rho^{*}\left(\sigma_{y} \otimes \sigma_{y}\right)$. After applying Eq. (3) with $\rho_{\mathcal{T}}=\rho_{\mathrm{I}}$, a straightforward calculation shows that there are only two nonzero terms in the decomposition of $\mathcal{M}_{\mathcal{T}}$ : namely, $\left\{\lambda_{0}=2(1-p), \lambda_{1}=p\right\}$, $\left\{T_{0}=\left(\begin{array}{ll}1 & 0\end{array}\right), T_{1}=\left(\begin{array}{c}0-1 \\ 0\end{array}\right)\right\}$. In physical terms, $T_{0}$ is a polarizer and $T_{1}$ is a $90^{\circ}$ polarization rotator. The two eigenvalues $\left\{\lambda_{0}, \lambda_{1}\right\}$ give the relative intensity in the two arms of the device and are physically realized by intensity attenuators.

\section{EXPERIMENT}

Our experimental setup is shown in Fig. 1. Its first part (singlet-state preparation) comprises a krypton-ion laser at $413.1 \mathrm{~nm}$ that pumps a 1 -mm-thick $\beta-\mathrm{BaB}_{2} \mathrm{O}_{4}$ (BBO) crystal, where polarization-entangled photon pairs at wavelength $826.2 \mathrm{~nm}$ are created by SPDC in a degenerate type-II phasematching configuration [22]. Single-mode fibers (SMFs) are used as spatial filters to assure that the initial two-photon state is in a single transverse mode. Spurious birefringence along the fibers is compensated by suitably oriented polarization controllers (PCs) [23]. In addition, total retardation introduced by the fibers and walk-off effects at the BBO 


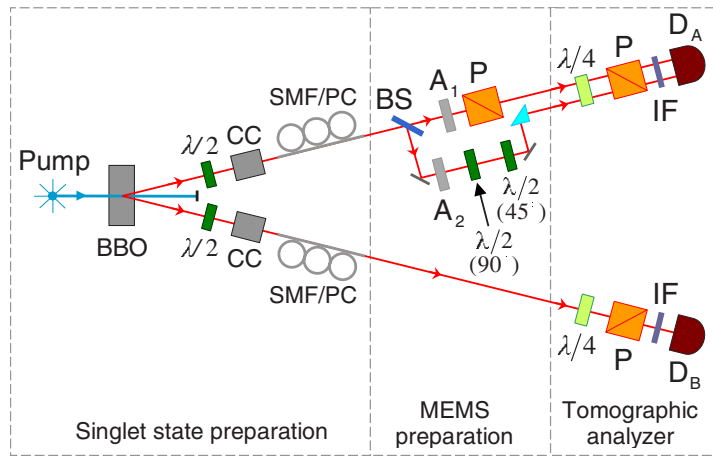

FIG. 1. (Color online) Layout of the experimental setup. The two-path optical device acts only on photon $A$. Detectors $\mathrm{D}_{\mathrm{A}}$ and $\mathrm{D}_{\mathrm{B}}$ perform coincidence measurements.

crystal are compensated for by compensating crystals (CCs: 0.5 -mm-thick BBO crystals) and half-wave plates $(\lambda / 2)$ in both photonic paths. In this way the initial two-photon state is prepared in the polarization singlet state $\left|\psi_{s}\right\rangle=(|H V\rangle$ $-|V H\rangle) / \sqrt{2}$, where $H(=0)$ and $V(=1)$ are labels for horizontal and vertical polarizations of the two photons, respectively.

In the second part of the experimental setup (MEMS preparation) the two-term decomposition of $\mathcal{M}_{\mathcal{T}}$ is physically realized by a two-path optical device. A photon enters such a device through a 50:50 beam splitter (BS) and can be either transmitted to path 1 or reflected to path 2 . The two paths defines two independent spatial modes of the field. In path 1 a neutral-density filter $\left(A_{1}\right)$ is followed by a linear polarizer (P) oriented horizontally (with respect to the BBO crystal basis). When the photon goes in this path, the initial singlet is reduced to $|H V\rangle$ with probability proportional to the attenuation ratio $a_{1}$ of $\mathrm{A}_{1}\left(a=P_{\text {out }} / P_{\text {in }}\right)$. In path 2 a second neutral-density filter $\left(\mathrm{A}_{2}\right)$ is followed by two half-wave plates $(\lambda / 2)$ in cascade relatively oriented at $45^{\circ}$ : they work as a $90^{\circ}$ polarization rotator. When the photon goes in path 2 , the singlet undergoes a local rotation with probability proportional to the attenuation ratio $a_{2}$ of $\mathrm{A}_{2}$. Note that the minimum coincidence rate is realized when either $a_{1}=0$ or $a_{2}=0$. However, even in these cases, detector $\mathrm{D}_{\mathrm{A}}$ has still $50 \%$ of probability to detect a photon and thus, at worst, half of the impinging photon intensity can be still detected.

The third and last part of the experimental setup (Tomographic analysis) consists of two tomographic analyzers (one per photon), each made of a quarter-wave plate $(\lambda / 4)$ followed by a linear polarizer $(\mathrm{P})$. Such analyzers permit a tomographically complete reconstruction, via a maximumlikelihood technique [24], of the two-photon state. Additionally, interference filters (IFs) in front of each detector $(\Delta \lambda=5 \mathrm{~nm})$ provide for bandwidth selection. It should be noticed that detector $\mathrm{D}_{\mathrm{A}}$ does not distinguish which path (either 1 or 2) a photon comes from; thus, photon $A$ is detected in a mode-insensitive way: This is the simple mechanism we use to induce decoherence. In the actual setup, a lens (not shown in Fig. 1) placed in front of detector $D_{A}$ focuses both paths 1 and 2 upon the sensitive area of the detector which becomes thus unable to distinguish between photons coming from either path 1 or 2 ("mode-insensitive detection").

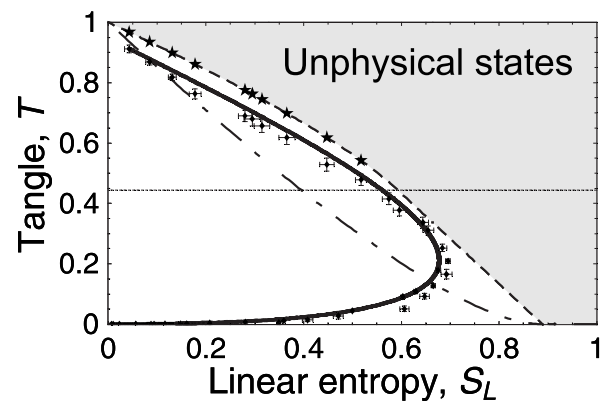

FIG. 2. Experimental data and theoretical prediction (solid line) in the linear entropy-tangle plane. The gray region represents unphysical states and it is bounded from below by MEMSs (dashed curve). The lower dot-dashed curve represents Werner states. The horizontal (dotted) line at $T=4 / 9$ separates MEMS I (above) from MEMS II (below). Stars denote MEMS I states $\rho_{\star}$ that have the same linear entropy as the measured states $\rho_{\mathrm{I}}^{\text {expt }}$ (i.e., the experimental points above the line $T=4 / 9)$. All measured data follow very well the theoretical curve.

Experimental results are shown in Fig. 2 together with theoretical predictions in the linear entropy-tangle plane. The agreement between theoretical predictions and measured data is very good. The experimentally prepared initial singlet state $\rho_{s}^{\text {expt }}$ has a fidelity [25] $\quad F\left(\rho_{s}, \rho_{s}^{\text {expt }}\right)=\left|\operatorname{Tr}\left(\sqrt{\sqrt{\rho_{s}} \rho_{s}^{\operatorname{expt}} \sqrt{\rho_{s}}}\right)\right|^{2}$ $\sim 97 \%$ with the theoretical singlet state $\rho_{s}$. The solid curve is calculated from the matrix $\rho_{c}: \rho_{c}=\mathcal{M}_{\mathcal{T}} \rho_{s}^{\text {expt }}$, and varying $p$. It represents our theoretical prediction for the given initially prepared state $\rho_{s}^{\text {expt }}$. If it were possible to achieve exactly $\rho_{s}^{\text {expt }}=\rho_{s}$, then such a curve would coincide with the MEMS curve above the horizontal (dotted) line $T=4 / 9$. Experimental points with $T \geqslant(4 / 9)\left(\rho_{\mathrm{I}}^{\text {expt }}\right)$ are obtained by varying the neutral-density filters $\mathrm{A}_{1}$ and $\mathrm{A}_{2}$ in such a way that $a_{2} \geqslant a_{1}$, while points with $T<4 / 9$ are achieved for $a_{2}<a_{1}$. Note that the latter points do not represent MEMSs, but different mixed entangled states whose density matrix is still given by $\rho_{\text {I }}$ but with the parameter $p$ now varying as $0 \leqslant p \leqslant 2 / 3$. The average fidelity between the measured states $\rho_{\mathrm{I}}^{\text {expt }}$ and the "target" states $\rho_{\star}$ is given by $\overline{F\left(\rho_{\star}, \rho_{\mathrm{I}}^{\text {expt }}\right)} \sim 80 \%$. The main reason for its deviation from $\lesssim 100 \%$ is due to spurious, uncontrolled birefringence in the BS and the prism composing the setup. To verify this, first we calculated the fidelity between the states $\rho_{c}(p)$ (obtained by applying the theoretically determined map $\mathcal{T} \otimes \mathcal{I}$ to the experimentally prepared initial singlet state $\rho_{s}^{\text {expt }}$, with the theoretical MEMS $\rho_{\mathrm{I}}(p)$. We have found $F\left[\rho_{\mathrm{I}}(p), \rho_{c}(p)\right] \geqslant 97 \%$ for all $2 / 3 \leqslant p \leqslant 1$; thus, the value of $\bar{F} \sim 80 \%$ cannot be ascribed to the imperfect initial singlet preparation. Second, we explicitly measured the Mueller matrices for both the BS and the prism (matrices that would be equal to the identity for ideal nonbirefringent elements) and we actually found spurious birefringence. From such measured matrices it was possible to determine the unwanted local unitary operation induced by these optical elements [26]. It is important to notice that such operation does not change the position of our experimental points in the linear entropy-tangle plane. Now, if one applies this unitary operation to our raw data and calculates once again the average fidelity, the result would be $\bar{F} \sim 91 \%$. 
However, since this "compensation" of the spurious birefringence is performed upon the measured data and not directly on the physical setup, we felt that it was more fair to present the uncorrected data.

\section{DISCUSSION AND CONCLUSIONS}

In conclusion, we have theoretically proposed and experimentally tested a simple method to create MEMS I states of photons. This method can be easily generalized to generate MEMS II states, as well. However, this task would require a slightly different experimental setup with a three-path linearoptical device acting only upon photon $A$ [26]. In particular, we have shown that it is possible to create a MEMS from a SPDC photon pair by acting on just a single photon of the pair. This task could appear, at first sight, impossible since it was recently demonstrated [14] that even the most general local operation cannot generate MEMSs because this would violate relativistic causality. However, as we discussed in the text, our results do not contradict Ref. [14] since we obtained them via post-selection operated by coincidence measurements. The latter are possible only when causal classical communication between detectors is permitted. Still, the connection between relativistic causality and dichroic (or PDL) devices and post-selection is far from being trivial. For example, suppose that a two-photon state is produced by an optical setup containing local PDL elements and that we tomographically reconstruct it after coincidence measurements. Such a reconstructed state will correctly describe the result of any other measurement involving coincidence measurements (such as, e.g., Bell measurements), but it will fail when describing the result of any single-photon measurement. We stress that this limitation is not inherent to our scheme, but it is shared by all optical setups containing PDL elements.

\section{ACKNOWLEDGMENTS}

We acknowledge Vladimir Bužek for useful correspondence. We also thank Fabio Antonio Bovino for helpful suggestions. This project is supported by FOM.
[1] M. A. Nielsen and I. L. Chuang, Quantum Computation and Quantum Information, reprinted first ed. (Cambridge University Press, Cambridge, UK, 2002).

[2] N. A. Peters, J. B. Altepeter, D. A. Branning, E. R. Jeffrey, T.-C. Wei, and P. G. Kwiat, Phys. Rev. Lett. 92, 133601 (2004).

[3] M. Barbieri, F. De Martini, G. Di Nepi, and P. Mataloni, Phys. Rev. Lett. 92, 177901 (2004).

[4] N. Brunner, A. Acín, D. Collins, N. Gisin, and V. Scarani, Phys. Rev. Lett. 91, 180402 (2003).

[5] A. Aiello, G. Puentes, D. Voigt, and J. P. Woerdman, Opt. Lett. 31, 817 (2006).

[6] J. N. Damask, Polarization Optics in Telecommunications (Springer, New York, 2005).

[7] D. L. Falkoff and J. E. McDonald, J. Opt. Soc. Am. 41, 862 (1951); U. Fano, Phys. Rev. 93, 121 (1954).

[8] M. Born and E. Wolf, Principles of Optics, 7th ed. (Cambridge University Press, Cambridge, England, 1999).

[9] A. Aiello and J. P. Woerdman, e-print arXiv:math-ph/0412061.

[10] S.-Y. Lu and R. A. Chipman, J. Opt. Soc. Am. A 13, 1106 (1996).

[11] M. B. Ruskai, S. Szarek, and E. Werner, Linear Algebr. Appl. 347, 159 (2002).

[12] D. G. M. Anderson and R. Barakat, J. Opt. Soc. Am. A 11, 2305 (1994).

[13] I. Bengtsson and K. Zyczkowski, Geometry of Quantum States. An Introduction to Quantum Entanglement (Cambridge University Press, Cambridge, England, 2006).
[14] M. Ziman and V. Bužek, Phys. Rev. A 72, 052325 (2005).

[15] In this article the symbols $\sigma_{x}, \sigma_{y}$, and $\sigma_{z}$ denote the three Pauli matrices as defined in J. J. Sakurai, Modern Quantum Mechanics (Benjamin/Cummings, Menlo Park, CA, 1985).

[16] The Hermitein, positive-semidefinite matrix $H$ was first introduced in polarization optics by R. Simon, Opt. Commun. 42, 293 (1982), and subsequently developed in Ref. [12]. It represents polarization-optics analogous to the dynamical (or Choi) matrix describing a one-qubit quantum process [E. C. G. Sudarshan, P. M. Mathews, and J. Rau, Phys. Rev. 121, 920 (1961); M.-D. Choi, Linear Algebr. Appl. 10, 285 (1975)].

[17] R. T. Thew and W. J. Munro, Phys. Rev. A 63, 030302(R) (2001).

[18] A. Aiello, G. Puentes, D. Voigt, and J. P. Woerdman, e-print arXiv:quant-ph/0603182.

[19] For sake of simplicity, we do not consider here type-II MEMSs. See, e.g., Ref. [2] and references therein.

[20] S. Bose and V. Vedral, Phys. Rev. A 61, 040101(R) (2001).

[21] W. K. Wootters, Phys. Rev. Lett. 80, 2245 (1998).

[22] P. G. Kwiat, K. Mattle, H. Weinfurter, A. Zeilinger, A. V. Sergienko, and Y. Shih, Phys. Rev. Lett. 75, 4337 (1995).

[23] G. Puentes, D. Voigt, A. Aiello, and J. P. Woerdman, Opt. Lett. 31, 2057 (2006).

[24] D. F. V. James, P. G. Kwiat, W. J. Munro, and A. G. White, Phys. Rev. A 64, 052312 (2001).

[25] R. Jozsa, J. Mod. Opt. 41, 2315 (1994).

[26] A. Aiello, G. Puentes, J. P. Woerdman, e-print arXiv:quant-ph/ 0611179. 